\title{
LA PROTECCIÓN DE LAS VÍCTIMAS DE TRATA. UN ENFOQUE INTEGRAL BASADO EN LOS DERECHOS HUMANOS
}

\author{
THE PROTECTION OF VICTIMS OF TRAFFICKING. AN INTEGRAL APPROACH BASED ON \\ HUMAN RIGHTS
}

\section{Isabel Ma Pérez Gázquez}

Prof. Dra. de Derecho del Trabajo y Seguridad Social. Universidad Católica San Antonio de Murcia.

E-mail: imperez@ucam.edu

Convidada

RESUMEN: La trata de seres humanos, cuyas principales víctimas son mujeres y niñas, constituye un atentando contra los valores superiores del ordenamiento jurídico y algunos de los derechos más básicos y fundamentales de las personas. Un abordaje eficaz de esta realidad exige la configuración de un amplio sistema de medidas de protección de las víctimas que ha de ir más allá de la esfera jurídica, e incluya una visión integral basada en la protección de los derechos humanos y las medidas preventivas. Este artículo tiene como objetivo examinar la complejidad y naturaleza del fenómeno de trata y los principales estándares y retos a los que nos enfrentamos en su lucha, desde una perspectiva que toma a la víctima como núcleo central de todas las medidas de protección

Palabras Clave: Trata de seres humanos. Víctimas de trata. Desamparo. Prevención. Integral

\begin{abstract}
Trafficking in human beings, whose mains victims are women and kids, constitutes an attack against the superior values of the legal system and some of the most basic and fundamental rights of people. An effective approach to this reality requires the configuration of a broad system of protection actions to protect victims that must go beyond the legal sphere, and include a comprehensive vision based on the protection of human rights and preventive actions. This article aims to examine the complexity and nature of the phenomenon of trafficking and the main standards and challenges that we face in their fight, from a perspective that takes the victim as the central nucleus of all protection measures.
\end{abstract}

Keywords: Traffiking in human beings. Victims. Helplessness. Prevention. Integral

SUMÁRIO: 1 El fenómeno de la trata de seres humanos. 1.1 Marco conceptual. 1.2 Factores que explican la trata. 2 Protección integral basada en los derechos humanos: la víctima como elemento central. 2.1 Elementos del enfoque integral basado en los derechos humanos. 2.1.1 Las tres "Ps" a) Prevención b) Protección c) Persecución de los tratantes. 3 Medidas de protección desde una perspectiva integral. La identificación de la víctima como elemento central. Conclusiones. Bibliografía. 


\section{EL FENÓMENO DE LA TRATA DE SERES HUMANOS}

\subsection{Marco conceptual}

La trata de seres humanos o también llamada esclavitud del S. XXI constituye un atentando contra algunos de los derechos más básicos y fundamentales de la persona como son la libertad, dignidad, e integridad física y emocional de la víctima. Se trata de un fenómeno en el que se produce la mercantilización y explotación de seres humanos que, a través de la interacción entre oferta y demanda, son utilizados como meros objetos para la obtención de un beneficio económico para los tratantes.

En este sentido, a través de actos como la captación; transporte; traslado; acogida o recepción, y distintos medios como la amenaza; el uso de la fuerza u otras formas de coacción; el rapto; el fraude; el engaño; el abuso de poder o de una situación de vulnerabilidad se produce la explotación de los seres humanos mediante diversas formas como son el trabajo forzado, la esclavitud, la prostitución, la servidumbre doméstica, el matrimonio forzado y la extracción de órganos ${ }^{1}$. Todo ello con el objetivo por parte de los tratantes de "exprimir" al máximo la rentabilidad económica de las personas explotadas, consideradas como productos, cuya consideración como seres humanos y el respeto que dicho status merece queda obviada y relegada por un interés económico.

Según lo dispuesto en el principio 8 de la Resolución de Naciones Unidas 60/147 aprobada por la Asamblea General el 16 de diciembre de 2005, sobre Principios y directrices básicos sobre el derecho de las víctimas de violaciones manifiestas de las normas internacionales de derechos humanos y de violaciones graves del derecho internacional humanitario a interponer recursos y obtener reparaciones, se considera víctima de trata a "toda persona que haya sufrido daños, individual o colectivamente, incluidas lesiones físicas o mentales, sufrimiento emocional, pérdidas económicas o menoscabo sustancial de sus derechos fundamentales, como consecuencia de acciones $\mathrm{u}$ omisiones que constituyan una violación manifiesta de las normas internacionales de derechos humanos o una violación grave del derecho internacional humanitario. Cuando corresponda, y en conformidad con el derecho interno, el término "víctima” también comprenderá a la familia inmediata o las personas a cargo de la víctima directa y a las personas que hayan sufrido daños al intervenir para prestar asistencia a víctimas en peligro o para impedir la victimización".

En definitiva, se trata de personas que, tras haber sido engañadas sobre el trabajo que van a realizar, viaje, o las condiciones de vida, entre otros aspectos, tienen una vida marcada por el abuso, la violación de derechos humanos y el constante control y coacciones por parte de sus captores, siendo una forma de explotación vinculada generalmente con la delincuencia organizada presente en varios países, por lo que se trata de una explotación y delito de carácter principalmente trasnacional.

A pesar de la dificultad de obtener datos exactos sobre este fenómeno -debido entre otros, a su vinculación con otros delitos, las distintas legislaciones nacionales y la falta de denuncia por parte de las propias víctimas, que en algunos casos ni siquiera son conscientes de estar siendo víctimas-, cabe reseñar que las estadísticas indican que, en el ámbito de los países miembros, las víctimas registradas a nivel mundial entre los períodos 2015-2016 fueron de 20.532, de las que más de la mitad (un $56 \%$ ) tenían por finalidad la explotación sexual, que continúa siendo la modalidad

\footnotetext{
${ }^{1}$ Artículo 3 del Protocolo para prevenir, reprimir y sancionar la trata de personas, especialmente mujeres y niños, que complementa la Convención de las Naciones Unidas contra la Delincuencia Organizada Transnacional, de 15 de noviembre de 2000 -Protocolo de Palermo-. Cabe reseñar su importancia en la delimitación y fijación de la concepción de trata de seres humanos. Hasta su aprobación, más allá de la prostitución, a nivel internacional no existía una definición clara y reconocida acerca de este fenómeno. Así, es a partir del mismo cuando se define el fenómeno de la trata como una situación de explotación de seres humanos que va más allá del ámbito sexual, incluyendo una amplia tipología de víctimas -hombres, mujeres y niños-.
}

Revista de Direito Brasileira | Florianópolis, SC | v. 28 | n. 11 | p.274-283 | Jan./Abr. 2021 
más extendida. En torno a una cuarta parte de los casos de trata $(26 \%)$ eran con fines de explotación laboral, y el resto (un $18 \%$ ) perseguía otros objetivos, tales como la mendicidad forzada o la extracción de órganos. Con respecto al perfil de las víctimas, señalar que mujeres y niñas constituyeron más de dos terceras partes $(68 \%)$ de las víctimas registradas. En cuanto a su nacionalidad, los cinco primeros países de procedencia de la UE fueron Rumania, Bulgaria, Países Bajos, Hungría y Polonia -esta lista no ha variado desde el período 2010-2012-, siendo Nigeria, China, Albania, Vietnam y Eritrea los cinco primeros países no integrantes de la UE ${ }^{2}$.

En suma, se trata de una realidad que atenta contra los derechos fundamentales de las personas y los valores superiores del ordenamiento jurídico, razón que exige el mayor de los esfuerzos y coordinación entre los distintos Estados e instituciones para establecer y llevar a cabo políticas y medidas eficaces que la disminuyan. En este sentido, son diversos los instrumentos que expresamente la regulan, prohíben y establecen medidas para su lucha ${ }^{3}$. No obstante, cabe tener en cuenta que la propia naturaleza de esta lacra y la falta de datos exactos en torno a la misma suponen una gran dificultad para el establecimiento de políticas públicas y medidas realmente efectivas.

En este contexto, el presente estudio examina la complejidad y naturaleza del fenómeno de trata y los distintos problemas y situaciones que envuelven a las víctimas desde una perspectiva que va más allá de la estrictamente jurídica. Así, se trata de exponer la realidad de este fenómeno desde la perspectiva de la víctima y de los derechos humanos, teniendo en cuenta su situación de vulnerabilidad y los distintos factores causantes, sobre los que resulta necesario intervenir.

\subsection{Factores que explican la trata}

Son diversos y de distinta naturaleza los factores que favorecen la producción de la situación de trata de seres humanos. Así, cabe diferenciar la existencia de factores relacionados con los tratantes, con la situación de las víctimas, y los países en los que se produce la captación y/o la explotación ${ }^{4}$

Entre los factores relacionados con los tratantes cabe destacar al factor económico y afán de lucro como elemento central. De este modo, dichos sujetos llevan a cometer tan grave y flagrante abuso de los derechos de otro ser humano tan sólo por una motivación de tipo económico. Asimismo, otro factor asociado a la producción de este abuso por parte de los tratantes es la falta de coordinación entre las distintas legislaciones nacionales y de persecución y sanción, que supone que la relación entre los riesgos y beneficios derivados de la explotación de otro ser humano resulte muy ventajosa para estos sujetos.

Por su parte, los factores relacionados con la víctima son de diversa naturaleza, si bien, todos tienen como común denominador la situación de vulnerabilidad, pobreza, y/o marginación. Así, los conflictos armados, inestabilidad política, los flujos migratorios, la falta de información y recursos, aspectos culturales, y la situación de miedo y desesperación que sufren algunas personas, entre otros, se convierten en factores de especial vulnerabilidad para éstas, que a su vez es

\footnotetext{
${ }^{2}$ COMISIÓN EUROPEA, Informe sobre los progresos realizados en la lucha contra la trata de seres humanos con arreglo al artículo 20 de la Directiva 2011/36/UE relativa a la prevención y lucha contra la trata de seres humanos y a la protección de las víctimas, 2016, págs. 2-3.

${ }^{3}$ En este sentido cabe reseñar al mencionado Protocolo de Naciones Unidas sobre la trata del año 2000 que complementan la Convención de las Naciones Unidas Contra la Delincuencia Transnacional Organizada (Protocolo de Palermo); Convenio del Consejo de Europa sobre la lucha contra la trata de seres humanos, firmado en Varsovia el 16 de mayo de 2005, entrada en vigor el 1 de febrero de 2008. Ratificado por España el 1 de abril de 2009 y entrado en vigor el 2 de agosto de.2009; Directiva 2011/36/UE relativa a la prevención y la lucha contra la trata de seres humanos y a la protección de las víctimas y por la que se sustituye la Decisión Marco 2002/629/JAI del Consejo; Convención de las Naciones Unidas sobre la Eliminación de todas las Formas de Discriminación contra la Mujer, de 18 de diciembre 1979.

${ }^{4}$ Véase, RUBIO LARA, P. A. Y PÉREZ ALBALADEJO, M., "El delito de trata de seres humanos en el derecho penal español: problemas e intentos de solución". Revista Aranzadi Doctrinal num.7, 2016, págs. 211-214.
} 
aprovechado por los tratantes. Así, éstos se aprovechan de las malas condiciones de vida de algunos países y la desesperación e instinto de supervivencia de las víctimas, que con la esperanza de alcanzar una vida mejor en otro país caen en las redes de dichos delincuentes.

Asimismo, cabe también tener en cuenta otros factores relacionados con la víctima que favorecen el mantenimiento de la situación de explotación tales como: las situaciones de coacción y perpetuación de la deuda por parte de los/as tratantes o intermediarios, las posibles situaciones de irregularidad administrativa en el país de explotación, el desconocimiento del idioma y cultura del país y/o la xenofobia por parte de la población del lugar al que llegan -en su caso-.

\section{PROTECCIÓN INTEGRAL BASADA EN LOS DERECHOS HUMANOS: LA VÍCTIMA COMO ELEMENTO CENTRAL}

En línea con el escenario expuesto, cabe decir que tradicionalmente el abordaje de este problema ha sido llevado a cabo tan sólo desde la perspectiva del Derecho Penal, a través del castigo para los tratantes. En este sentido, es necesario advertir que, si bien este tipo de medidas de castigo son necesarias, por sí solas no resultan suficientes para la resolución de esta realidad. Asimismo, su aplicación sin otras medidas de protección puede producir otros perjuicios para la víctima, que constituirían lo que se ha venido a llamar como fenómeno de victimización secundaria.

De esta manera, los daños colaterales que se pueden producirse para la víctima pueden venir derivados de su detención en centros de inmigración, -al considerarles inmigrantes en situación irregular en lugar de víctimas de trata-, el enjuiciamiento en delitos conexos a la situación de trata y que son ajenos a su voluntad, y/o su repatriación forzosa a los países de origen sin garantías y sin tener en cuenta el peligro de sufrir represalias, entre otros ${ }^{5}$.

Ante este contexto de ineficacia de este tipo de medidas para reducir los casos de trata, se fue abriendo paso la concepción de un abordaje integral -desde la perspectiva de la víctima y de los diversos factores que favorecen su captación- que incluye medidas específicas de protección frente a las consecuencias del citado fenómeno de victimización secundaria derivados de la estricta aplicación del derecho penal. Así, fue especialmente a partir del informe del Alto comisionado de Naciones Unidas para los Derechos Humanos, de 20 de mayo de 2002, sobre Principios y Directrices recomendados sobre los derechos humanos y la trata de personas, el Convenio del Consejo de Europa sobre trata de 2005 (Convenio de Varsovia) y la Directiva UE 2011/36 relativa a la prevención y la lucha contra la trata de seres humanos y a la protección de las víctimas y por la que se sustituye la Decisión Marco 2002/629/JAI del Consejo (Directiva sobre trata) cuando comenzaron a tomarse medidas desde una la perspectiva del Derecho Internacional y los Derechos Humanos, que toma como referencia central a la víctima.

Es importante reseñar la intervención del Tribunal Europeo de Derechos Humanos (TEDH), cuya interpretación extensiva del artículo 4 del Convenio para la protección de los Derechos Humanos y de las Libertades Fundamentales, de 4 de noviembre de 1950, que establece la prohibición de la esclavitud y trabajo forzoso, incluye a la trata de personas bajo el marco protector del Derecho Internacional, cuyas consecuencias, entre otras, incluye la obligatoriedad de que los Estados luchen contra la trata a través de la prevención, la investigación y el enjuiciamiento $^{6}$. Todo ello, teniendo en cuenta que no sólo se obliga a los Estados al establecimiento de medidas integrales de protección, a las que seguidamente se hará mención, sino

\footnotetext{
${ }^{5}$ MILANO V., "Protección de las víctimas de trata con fines de explotación sexual: estándares internacionales en materia de enfoque de derechos humanos y retos relativos a su aplicación en España”. Revista electrónica de estudios internacionales, 32/2016, DOI: 10.17103/reei.32.05, págs. 5-13.

${ }^{6}$ TEDH, Rantsev c. Chipre y Rusia (n. 25965/04), de 7 de enero de 2010 (Rantsev c. Chipre y Rusia), pár. 285. Para mayor abundamiento acerca de esta sentencia y sus consecuencias véase, LUCEA SÁENZ, A., "La lucha contra la trata de seres humanos en la Unión Europea". Aequalitas: Revista jurídica de igualdad de oportunidades entre hombres y mujeres, núm. 32, 2013, págs. 6-15.
}

Revista de Direito Brasileira | Florianópolis, SC | v. 28 | n. 11 | p.274-283 | Jan./Abr. 2021 
que además se les obliga a actuar bajo los parámetros del deber de diligencia, es decir, bajo unos estándares mínimos ${ }^{7}$.

Se produjo, por tanto, el tránsito de las medidas sancionatorias penales, centradas exclusivamente en los factores de los tratantes, a un tratamiento más amplio e integral, basado en la protección de todas las esferas de la víctima, cuyo objetivo es la evitación de la situación de explotación y la protección de los derechos humanos ${ }^{8}$.

Son diversas las ventajas que se han señalado con respecto a este enfoque victimocéntrico y no meramente criminalístico, entre ellas que: "promueve una mejor comprensión de los problemas experimentados por las personas objeto de trata. Las personas objeto de trata pueden ser vistas como víctimas de abusos a los derechos humanos en lugar de criminales que violan las leyes y regulaciones nacionales de inmigración (...). Asimismo, "permite abordar cuestiones más amplias (...), incluidas las causas y consecuencias, que deben abordarse para prevenir y reprimir eficazmente el fenómeno. Un marco de derechos humanos nos permite entender estos temas en profundidad y buscar soluciones no solo legales, sino también políticas, económicas y sociales en consecuencia. En otras palabras, tiene el potencial de promover un enfoque holístico y, por lo tanto, fortalecer la acción global contra el fenómeno" $"$.

\subsection{Elementos del enfoque integral basado en los derechos humanos}

Según lo anterior, se produjo el inicio de un nuevo enfoque basado en la protección de las víctimas de trata a través de un abordaje holístico e integral basado en la primacía de los derechos humanos de la víctima bajo la perspectiva de las tres "Ps". Esto es, prevención, protección, persecución y debida diligencia por parte del Estado ${ }^{10}$.

Así, se inició un cambio de rumbo hacia una perspectiva que parte de la consideración de la víctima como el elemento central de todas las medidas a llevar a cabo por el Estado, considerándola como un todo, es decir, como un ser humano cuya protección requiere, además de medidas jurídicas, otras de distinta naturaleza, como son las de tipo psicosocial y preventivo.

De este modo, todas las medidas llevadas a cabo contra la trata han de tener como objetivo principal, además del castigo de los tratantes y el control del flujo migratorio, el bienestar y protección de los derechos de las víctimas ${ }^{11}$.

\subsubsection{Las tres "P"}

\section{a) Prevención}

Desde la perspectiva de la prevención se hace necesario el estudio de los factores y problemas de base que llevan a las víctimas de trata a encontrarse en la situación de vulnerabilidad, y que como ya se señaló atiende a distintas situaciones cuyo denominador común es la situación de exclusión, marginación y pobreza.

\footnotetext{
7 COMITÉ PARA LA ELIMINACIÓN DE LA DISCRIMINACIÓN CONTRA LA MUJER (CEDAW), 2010, Recomendación general núm. 19, 1992, pár. 9 y Recomendación general núm. 28, 2010, pár. 13.

${ }^{8}$ TEDH, Rantsev c. Chipre y Rusia (n. 25965/04), de 7 de enero de 2010 (Rantsev c. Chipre y Rusia), pár. 285.

${ }^{9}$ OBOKATA, T., "A Human Rights Framework to Address Trafficking of Human Beings [Un marco de derechos humanos para abordar la trata de seres humanos]". Netherlands Quarterly of Human Rights, núm. 24, 2016, p. 379, doi.org/10.1177/016934410602400302.

${ }^{10}$ Véase, RODRÍGUEZ RODRÍGUEZ, F.M., "Los derechos humanos de las víctimas de trata con la finalidad de explotación sexual". Cuadernos Electrónicos de Filosofía del Derecho, núm. 39, 2019, págs. 94-112; MILANO V., "Protección de las víctimas de trata con fines de explotación sexual...ob. cit. págs. 13-28.

${ }^{11}$ Convenio de Varsovia, preámbulo, considerando 4; COMISIÓN EUROPEA, Comunicación al Parlamento europeo, al Consejo, al Comité Económico y Social y al Comité de las Regiones, Estrategia de la UE para la erradicación de la trata de seres humanos (2012 -2016), Bruselas, 19 de junio de 2012, pág. 6.
} 
Esta situación de necesidad, unida a otros factores como pueden ser el deseo de migración a otro país, la falta de redes de apoyo, recursos o falta de información, se convierten en elementos de fuerza para los explotadores ya que, ante la expectativa de una vida mejor y la posible promesa por parte de los tratantes de un trabajo, o la ayuda para cruzar las fronteras, estas personas acaban en las redes de los tratantes.

En este sentido, son diversas las medidas de tipo preventivo específicamente reguladas por distintos instrumentos normativos. Así, se regula la necesidad de establecer programas y políticas públicas de prevención orientadas a la información, las investigaciones, la información, las campañas de sensibilización y educación, las iniciativas sociales y económicas y los programas de formación dirigidos a las personas vulnerables a la trata de seres humanos y a los profesionales que trabajan en este ámbito. Asimismo, se establecen medidas para desincentivar la demanda de los servicios de la trata bajo el razonamiento de que reduciendo la demanda se reducirían los beneficios económicos de los tratantes y, por tanto, dejaría de ser rentable la explotación de las personas. De igual modo, se prevén medidas de control en los puestos fronterizos ${ }^{12}$.

\section{b) Protección}

Las medidas llevadas a cabo desde la óptica integral han de estar orientadas a evitar las ya citadas situaciones de segunda victimización que en algunos casos se produce en las víctimas, así como a dar una protección y asistencia adecuada. Así, referido a la evitación de las situaciones de segunda victimización, el Estado está obligado a luchar contra la trata sin producir nuevamente la vulneración de los derechos de la víctima.

Por otro lado, en lo relativo a la prestación de una asistencia realizada bajo los parámetros del deber de debida diligencia, supone la obligación de prestar todos los medios y asistencia necesarios desde un punto de vista integral, es decir, abordando y dando respuesta a todas las necesidades y problemáticas en torno a la situación de explotación, que además han de realizarse de acuerdo con el perfil de la víctima, teniendo en cuenta que en el caso de los menores resulta precisa la adopción de un mayor número de medidas protectoras. Así, resulta necesario el establecimiento de medidas de diverso tipo y naturaleza, a las que se hará mayor mención en las siguientes páginas ${ }^{13}$.

\section{c) Persecución de los tratantes}

Por último, la última "P" hace referencia al establecimiento, también necesario y fundamental junto a las anteriores medidas, de las clásicas medidas punitivas y sancionatorias del Derecho Penal para condenar y castigar a los tratantes ${ }^{14}$.

\footnotetext{
${ }^{12}$ Convenio del Consejo de Europa sobre la lucha contra la trata de seres humanos, firmado en Varsovia el 16 de mayo de 2005, arts. 5-19; Naciones Unidas., Resolución 60/147 de 16 de diciembre de 2005, sobre principios y directrices básicos sobre el derecho de las víctimas de violaciones manifiestas de las normas internacionales de derechos humanos y de violaciones graves del derecho internacional humanitario a interponer recursos y obtener reparaciones, principios 4 a 6 y directrices 1, 3, 7, 10 y 11; Directiva 2011/36/UE relativa a la prevención y la lucha contra la trata de seres humanos y a la protección de las víctimas y por la que se sustituye la Decisión Marco 2002/629/JAI del Consejo, artículos 18 y 23.2 .

${ }^{13}$ Ibídem, artículos 11-12 y 17; principios 6-8; art. 11, respectivamente.

${ }^{14}$ Véase art. 177 bis del Código Penal, incorporado por la Ley Orgánica 5/2010 por la que se modifica la Ley Orgánica 10/1995, de 23 de noviembre. Para mayor abundamiento consúltese RUBIO LARA, P. A. Y PÉREZ ALBALADEJO, M., "El delito de trata de seres humanos... ob. cit. págs. 207-250.
} 


\subsection{Medidas de protección desde una perspectiva integral. La identificación de la víctima como elemento central}

Son numerosas y de diverso tipo las acciones llevadas a cabo para proteger a las víctimas de trata de seres humanos, entre las que, cada vez más, adquieren mayor protagonismo las orientadas a la prevención y protección, con medidas de tipo psicosocial, sanitario, educativo, jurídico, de subsistencia (alojamiento, acceso al mercado laboral), integrador en la sociedad.

Asimismo, se prevén el establecimiento de otras medidas como son el período de reintegración y reflexión de al menos treinta días para aquellas personas sobre las que existan sospechas de ser víctima de trata; la obtención de un permiso de residencia para los casos en los que la colaboración de la víctima sea necesario para el desarrollo de las actuaciones e investigaciones penales o sea necesario por su situación personal; la posibilidad de recibir una indemnización y el establecimiento de medidas que permitan la identificación de la víctima ${ }^{15}$.

Ahora bien, resulta preciso tener en cuenta que el elemento central y delimitador de la eficacia de tales medidas es la identificación de las víctimas ${ }^{16}$. Piénsese que de nada serviría un amplio protocolo, con un gran elenco de medidas, si no se es capaz de identificar a los sujetos a los que han de ir destinadas. Así, el elemento principal de las medidas de protección se relaciona íntimamente con una de las medidas de prevención, la capacitación y formación adecuada de los profesionales implicados en los ámbitos en los que pueda existir una interacción con la víctimas de $\operatorname{trata}^{17}$.

La identificación de las víctimas es llevada a cabo principalmente por investigaciones realizadas por los Cuerpos y Fuerzas de Seguridad del Estado, ya sea a través de medidas de control en puestos fronterizos o en otro tipo de intervenciones policiales. Asimismo, también pueden llevarse a cabo a través de inspecciones de trabajo o del contacto de la víctima con otras instituciones como consecuencia del acceso a asistencia sanitaria, educativa o social, o a través de dispositivos de información (unidades móviles o servicios de atención telefónica), entre otros, en los que la valoración de ciertos indicadores resulta imprescindible para llevar a cabo una adecuada y eficaz detección ${ }^{18}$.

Si bien es cierto que estos indicadores por sí solos no son suficientes para afirmar la existencia de una situación de trata de seres humanos, si deben ser tomados en cuenta para profundizar en dicha situación. Téngase en cuenta que en la gran mayoría de los casos de explotación la víctima tiende a esconder su situación -ya sea por el estigma social que ello implica o por miedo a posibles represalias contra ellas o contra sus familias de origen-. Cuestión que, a su vez, exige la adecuada y especializada capacitación y formación de los profesionales implicados en los ámbitos en los que pueda existir una interacción con estas víctimas ${ }^{19}$.

La propia complejidad y diversidad del fenómeno de la trata impide establecer una fórmula o parámetros únicos que la definan. No obstante, y a pesar de que cada víctima puede tener una experiencia distinta, existen ciertos comportamiento o situaciones que -en mayor o menor medida- resultan comunes y, por tanto, son considerados indicadores de una posible situación de explotación de seres humanos. De este modo, cabe tener en cuenta indicadores de distinta naturaleza como son los relativos a la salud, lugar de origen y rutas relacionadas con situaciones de trata, situación sociocultural y educativa, ausencia de contacto familiar o social, incapacidad de

\footnotetext{
${ }^{15}$ Ibídem, Artículos 10 y 13-16; principio 9; art 11, respectivamente.

${ }^{16}$ VILLACAMPA ESTIARTE, C. Y TORRES ROSELL, N., "Protección jurídica y asistencia para víctimas de trata de seres humanos". Revista General de Derecho Penal, núm. 27, 2017.

17 CABRERA RODRÍGUEZ, E., "La trata de seres humanos con fines de explotación sexual. La detección e identificación de las víctimas". Revista de derecho y proceso penal, núm. 45, 2017, págs. 97-115.

${ }^{18}$ MINISTERIO DE IGUALDAD., Protocolo marco de protección de las víctimas de trata de seres humanos del Ministerio de Sanidad, Servicios sociales e igualdad, 2011.

19 CABRERA RODRÍGUEZ, E., "La trata de seres humanos con fines de explotación sexual. La detección e identificación de las víctimas". Revista de derecho y proceso penal, núm. 45, 2017, págs. 97-115.
} 
comunicación, o lugares en los que presuntamente existen mayores posibilidades de esta situación de explotación (fuerzas y cuerpos de seguridad, psicólogos, sanitarios, educadores, inspectores de trabajo y seguridad social, entre otros) ${ }^{20}$.

Relativos a la salud conviene reseñar situaciones relacionadas tanto con la salud física de la posible víctima (desnutrición, infección y enfermedades de transmisión sexual, moratones o lesiones que no han sido médicamente tratados, entre otros) como con la psicológica (trastornos psicológicos como ansiedad, fobias, estrés agudo, pánico, depresión, problema de adicción, cuya sintomatología más frecuente suele estar relacionada con: dolores de cabeza, alteraciones del sueño, cansancio generalizado, apariencia nerviosa o preocupada, facilidad de llanto, dificultad y/o lentitud de pensamiento, pérdida de interés o autoestima, entre otros). En lo que se refiere a las menores, además de los indicadores señalados, cabe tener también en cuenta aspectos como una baja estatura o desarrollo acorde a su edad -que podrían indicar situaciones de desnutrición-, problemas de desarrollo cognitivo -manifestado por dificultades en el habla o aprendizaje, fracaso escolar-, o problemas de tipo conductual como conocimientos sexuales no acordes a la edad ${ }^{21}$.

De igual modo, cabe considerar como indicios determinados lugares tales como clubes nocturnos o de masaje, talleres clandestinos, residencias habitacionales colectivas, centros de internamiento de inmigrantes irregulares, entre otros, en los que es posible encontrar potenciales víctimas de trata y sobre los que tanto la sociedad como las distintas instituciones públicas no parecen reparar.

Por último, cabe hacer mención a que uno de los principales problemas en torno a la situación de detección de la situación de explotación es la confusión que a veces se produce entre la situación de migración irregular y las víctimas de trata. Así, se hace necesario tener en cuenta que, si bien es cierto que gran número de las víctimas de trata son inmigrantes irregulares, ni todas ellas se encuentran en dicha situación, ni toda la migración irregular es víctima de trata.

\section{CONCLUSIONES}

Aunque es innegable reconocer la producción de grandes avances en cuanto a la protección de esta lacra, la encomiable labor que tanto las fuerzas y cuerpos de seguridad del Estado e instituciones implicadas realizan en su lucha, y el aumento del número de protocolos y planes de actuación que tratan el problema desde un punto de vista de protección de los derechos humanos, que va más allá de la mera persecución de los tratantes, aún queda mucho por avanzar, especialmente en lo que se refiere a los aspectos preventivos desde la educación e información. Es por ello, que resulta necesario el establecimiento de acciones formativas y de capacitación de todos los profesionales y agentes implicados en las distintas situaciones que rodean a la situación de trata de personas. De igual modo, es preciso dar mayor fomento a la realización de campañas de información y sensibilización de los ciudadanos acerca de esta realidad, con el objetivo de concienciar acerca de la grave violación de derechos fundamentales que conllevan estas situaciones de explotación, y de la necesidad de condena por parte de todos los individuos como vía para la disminución o eliminación de este fenómeno. Asimismo, se hace también necesario realizar campañas de información acerca de esta realidad, en este caso, a aquellas personas en situación de vulnerabilidad y riesgo de ser víctima de trata, con información acerca de los posibles indicadores, formas y vías de denuncia y salida de dicha situación.

\footnotetext{
20 Véase, MINISTERIO DEL INTERIOR., Trata de seres humanos. Recuperado de: https://www.policia.es/_es/colabora_trata.php. Consultado: 12 de abril de 2021.

${ }^{21}$ CODERO RAMOS, N. Y FERNÁNDEZ ESQUIVEL, C., "Trabajo Social Hospitalario con mujeres subsaharianas posibles víctimas de trata”. Revista Agathos. Atención sociosanitaria y bienestar, núm. 2, 2016, págs.28-37.
} 


\section{BIBLIOGRAFÍA}

CABRERA RODRÍGUEZ, E., "La trata de seres humanos con fines de explotación sexual. La detección e identificación de las víctimas". Revista de derecho y proceso penal, núm. 45, 2017 , págs. 97-115.

CODERO RAMOS, N., ANTOLINEZ DOMÍNGUEZ, I. Y JORGE BARBUZANO, E., "Intervención social con menores posibles víctimas de trata. Niñas y niños que cruzan fronteras", en AAVV. La intervención social con menores, Cordero Ramos, N. y Nieto Morales, C, (Dirs.), Ed. Dykinson, Madrid, 2016, págs. 226-243.

CODERO RAMOS, N. Y FERNÁNDEZ ESQUIVEL, C., “Trabajo Social Hospitalario con mujeres subsaharianas posibles víctimas de trata". Revista Agathos. Atención sociosanitaria y bienestar, núm. 2, 2016, págs.28-37.

COMISIÓN EUROPEA. Segundo informe sobre los progresos realizados en la lucha contra la trata de seres humanos (2018) con arreglo al artículo 20 de la Directiva 2011/36/UE relativa a la prevención y lucha contra la trata de seres humanos y a la protección de las víctimas.

COMISIÓN EUROPEA. Estrategia de la UE para la erradicación de la trata de seres humanos (2012 -2016), Bruselas, 19 de junio, 2012.

\section{COMITÉ PARA LA ELIMINACIÓN DE LA DISCRIMINACIÓN CONTRA LA MUJER} (CEDAW, 2010).

CONSEJO DE EUROPA., Convenio sobre la lucha contra la trata de seres humanos, firmado en Varsovia el 16 de mayo de 2005.

DEFENSOR DEL PUEBLO. La trata de seres humanos en España: victimas invisibles, 2012. Recuperado de https://www.defensordelpueblo.es. Consultado: 1/11/2019.

DELGADO SANCHO, C., D., "La trata de seres humanos y la inmigración clandestina tras la reforma de la Ley orgánica 1/2015”. Revista Aranzadi Doctrinal, núm. 8, 2017, págs. 253-271.

LUCEA SÁENZ, A., "La lucha contra la trata de seres humanos en la Unión Europea". Aequalitas: Revista jurídica de igualdad de oportunidades entre hombres y mujeres, núm. 32, 2013, págs. 6-15.

MILANO V., "Protección de las víctimas de trata con fines de explotación sexual: estándares internacionales en materia de enfoque de derechos humanos y retos relativos a su aplicación en España". Revista electrónica de estudios internacionales, núm. 32, 2016, doi:10.17103/reei.32.05.

MINISTERIO DE SANIDAD, SERVICIOS SOCIALES Y SANIDAD. Plan integral de lucha contra la trata de mujeres y niñas con fines de explotación sexual para los años 2015-2018. 
MINISTERIO DE IGUALDAD. Protocolo marco de protección de las víctimas de trata de seres humanos del Ministerio de Sanidad, Servicios sociales e igualdad, 2011.

MINISTERIO DEL INTERIOR., Trata de seres humanos. Recuperado de: https://www.policia.es/_es/colabora_trata.php. Consultado: 12 de abril de 2021.

MINISTERIO DEL INTERIOR., Instrucción 6/2016, de la Secretaría de Estado y Seguridad, sobre actuaciones de las Fuerzas y Cuerpos de Seguridad del Estado en la lucha contra la trata de seres humanos y en la colaboración con las organizaciones y entidades con experiencia acreditada en la asistencia a las víctimas.

NACIONES UNIDAS., Protocolo para prevenir, reprimir y sancionar la trata de personas, especialmente mujeres y niños, que complementa la Convención de las Naciones Unidas contra la Delincuencia Organizada Transnacional, de 15 de noviembre, de 2000.

NACIONES UNIDAS., Resolución 60/147 sobre Principios y directrices básicos sobre el derecho de las víctimas de violaciones manifiestas de las normas internacionales de derechos humanos y de violaciones graves del derecho internacional humanitario a interponer recursos y obtener reparaciones, de 16 de diciembre, de 2005.

OBOKATA, T., "A Human Rights Framework to Address Trafficking of Human Beings [Un marco de derechos humanos para abordar la trata de seres humanos]". Netherlands Quarterly of Human Rights, núm. 24, 2016, doi.org/10.1177/016934410602400302.

PARLAMENTO EUROPEO., Informe sobre la lucha contra el tráfico de seres humanos en las relaciones exteriores de la UE (2015/2340(INI)), de 13 de junio, de 2016.

RODRÍGUEZ RODRÍGUEZ, F.M., "Los derechos humanos de las víctimas de trata con la finalidad de explotación sexual". Cuadernos Electrónicos de Filosofía del Derecho, núm. 39, 2019, págs. 94-112.

RUBIO LARA, P. A. Y PÉREZ ALBALADEJO, M., "El delito de trata de seres humanos en el derecho penal español: problemas e intentos de solución”. Revista Aranzadi Doctrinal, núm. 7, 2016, págs. 207-250.

VILLACAMPA ESTIARTE, C. Y TORRES ROSELL, N., "Protección jurídica y asistencia para víctimas de trata de seres humanos”. Revista General de Derecho Penal, núm. 27, 2017, págs. 1698-1189.

VILLACAMPA ESTIARTE, C., "La nueva directiva europea relativa a la prevención y la lucha contra la trata de seres humanos y a la protección de las víctimas. ¿Cambio de rumbo de la política de la Unión en materia de trata de seres humanos?’. Revista Electrónica de Ciencia Penal y Criminología, núm. 13, 2011. 